\title{
Comparative study of the in-situ and laboratory estimated and measured unsaturated hydraulic properties of soil under different conditions
}

\author{
Gamal ABOUZEID ${ }^{1}$, Tai SASAKI ${ }^{2}$, Kunio WATANABE ${ }^{3}$, \\ Mohamed ASHOUR ${ }^{4}$, Nashaat ALY ${ }^{4}$, and Mohamed ALY 4 \\ ${ }^{1}$ Member of JSCE, Egyptian Ph. D. Channel System Student, Civil Eng. Dept., Saitama Univ. \\ 2 Member of JSCE, Civil \& Architectural Engineering Dept., Japan Nuclear Fuel Limited. \\ ${ }^{3}$ Member of JSCE, Prof, Civil Eng. Dept., Saitama Univ. 255 Shimo-Okubo, Urawa-Shi, Saitama 338, Japan. \\ ${ }^{4}$ Egyptian supervisors of the Ph.D. Channel system student, Civil Eng. Dept., Assiut Univ., Assiut, Egypt.
}

\begin{abstract}
Accurate descriptions of soil hydraulic properties are essential to predict the behavior of water and contaminants in the unsaturated zone. A new technique was used to estimate the unsaturated hydraulic properties of volcanic ash soil which is one of the composition of Quaternary layer distributed around the location of disposal facility at Rokkasho low level radioactive waste disposal site, Japan. A comparison between the measured and estimated unsaturated hydraulic properties of this type of soil and also with the type of volcanic ash found in Saitama University and in the research institute of agricultural environment, Tuskuba, namely by Kanto-loam. It was found from the physical and hydraulic properties that, the volcanic ash in Rokkasho has low hydraulic conductivity and high capillary head, also the laboratory test on undisturbed soil specimen can represent the actual field properties with the proposed technique.
\end{abstract}

\section{Keywords: Unsaturated Hydraulic Conductivity, Capillary Head, Evaporation, Saturation distribution}

\section{Introduction}

The increasing concern with groundwater pollution and contamination of soils by hazardous substances has stimulated the development of numerous mathematical models of pollutant transport in soils. Today, the numerical simulation of water and pollutant transport in unsaturated soils has become a standard tool for assessing environmental pollution problems. Many options exist for including hydraulic properties in models: tabular data, linked polynomials, summed functions, and analytical functions [e.g., Ross et al., 1991; Ross and Smettem, 1993; Durner, 1994 and Rossi and Nimmo, 1994]. By far the most commonly used retention function is that proposed by van-Genuchten (1980) combined with Mualem's conductivity model (1976) to describe unsaturated hydraulic conductivity. This models is popular because it easily fits water retention data in the wet region where most flow occurs, and have a minimum number of parameters that allow for easy description. Numerous laboratory and field methods for direct measurement as well as estimation methods are available to predict the unsaturated hydraulic properties of soil. The most obvious way to obtain these properties is by experiments; however, these tend to be difficult, laborious, and time consuming. Moreover, available methods are limited in range of water content, and they required sophisticated equipment or extensive preparation. Hence, In the present study, an effort has been made to develop an in-situ and laboratory new technique for creating unsaturated water flow through soil core using new evaporation measurement equipment. Numerically and on the basis of Galerkin approach in the finite element method, the unsaturated flow equation was inversely solved. The van-Genuchten model (1980) was adopted to represent the unsaturated hydraulic properties of the soil. Unknown parameters in this model were estimated from the observed vertical saturation profile and numerical inversion of unsaturated water flow equation. The applicability of this technique was successfully checked by some insitu tests. A comparison has been made between the measured and estimated unsaturated hydraulic conductivity and capillary pressure for the same soil type and between the studied two types of volcanic ash. Also, the results obtained from laboratory technique were quantitatively compared with those obtained from the in-situ one for the same soils to test the validity of laboratory test and check the effect of soil disturbance on its properties. 


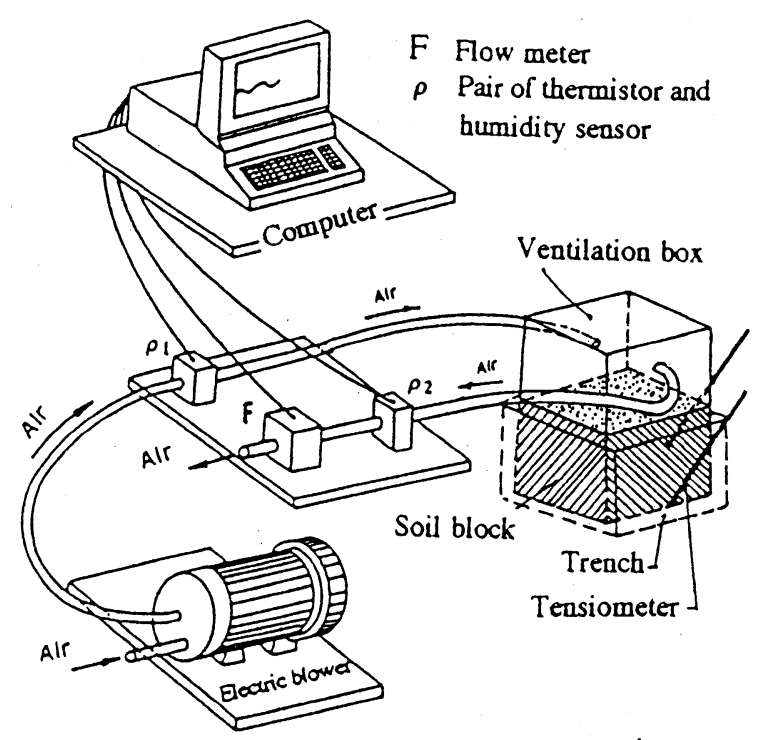

Fig. 1 In-situ soil block and evaporation measurement equipment

\section{Basic Idea of The Unsaturated Hydraulic Properties Estimation Technique}

The hydraulic properties estimation technique proposed here is composed of two parts. The first is an in-situ or laboratory test for making well-defined unsaturated flow in a tested soil core. The boundary conditions are controlled to make a simple flow through this soil core. Saturation distribution in this core is measured after the test. The second part of this technique is the inverse solution of the unsaturated flow in the tested soil core for estimating the hydraulic properties. The hydraulic properties are mathematically looked for by reconstructed the measured saturation distribution. Many calculations with changing the properties are needed in this technique.

\section{Experimental Measurements}

Techniques

and

Figure 1 schematically shows the in-situ test. As shown in this figure, a separated soil block $(60 \mathrm{~cm}$ long, $50 \mathrm{~cm}$ wide and $45 \mathrm{~cm}$ deep) was prepared by excavating a trench around the block. A plastic sheet for stopping the water flow through these sides wrapped the sidewalls of this block, and then the trench was filled with soil. A tensiometer was fixed at the bottom of this block for measuring the capillary pressure as a bottom boundary head condition. Another tensiometer was installed at a depth of $18 \mathrm{~cm}$ to check a head. When the air humidity above the top surface of the block is low, water is evaporated through this surface and water contained in this soil block upwardly flowing, then an upward unsaturated flow was formed in this block. Evaporation rate at the top surface of this block was measured for getting the

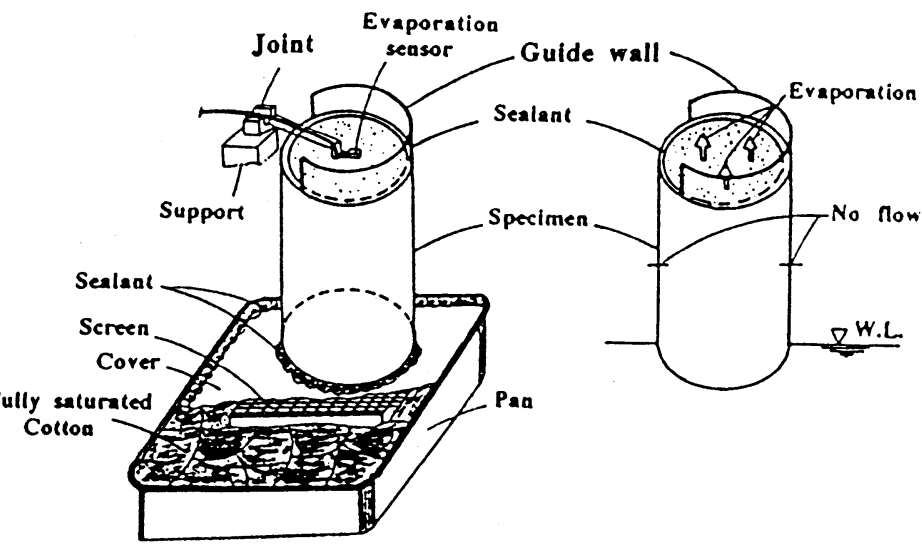

Fig. 2 Laboratory test and boundary condition

surface flux condition. The vertical saturation distributions could be measured at the beginning and at the end of the test respectively by collecting soil samples from the soil block and gravitational method was used.

The in-situ measurements of the water flow rate in unsaturated soils and other flow parameters thought to be difficult, a new evaporation measurement technique which has been proposed by Watanabe et al., (1994) was used for measuring the evaporation rate from the top surface of the soil block. This technique depends on the fact that if some part of the ground surface is covered by a box made from the transparent sheets and the air is injected from one side and exhausted from the opposite side, the absolute humidity of the exhausted air increases when the vapor is coming out from the ground surface by the evaporation. Air was injected into the ventilation box (Fig. 1), and exhausted from the other side through two pipes attached on both sidewalls using blower system. The flow rate of passing air through this box was measured by a flow meter F. The temperatures and relative humidity of injected and exhausted air were continuously measured by a couple of thermistor and humidity sensors $\rho$ 1,2 (Watanabe, 1991). The evaporation rate $\mathrm{Ev}$ in $\mathrm{g} / \mathrm{cm} 2 / \mathrm{sec}$ from this surface could be calculated by the following equation;

$$
E v=q(\lambda 2-\lambda 1) / A
$$

where, $\mathrm{q}$ is air flow rate in $\mathrm{cm} 3 / \mathrm{sec}, \lambda$ is absolute humidity of air in $\mathrm{g} / \mathrm{cm} 3$ (calculated from relative humidity and temperature ), and $\mathrm{A}$ is area covered by ventilation box in $\mathrm{cm} 2$. The subscripts 1 and 2 are representing the injected and the exhausted air, respectively. 
In case of laboratory test, considering same idea for creating unsaturated flow in the in-situ test, unsaturated flow could be created in a small well packed undisturbed and/or disturbed soil specimen. As shown in Fig. 2, the sidewall of soil specimen is completely sealed to prevent the evaporation from this wall surface. A constant pressure head is given on the bottom by putting the specimen on a pan filled with continuos fully saturated cotton. When the air humidity above the top surface of this specimen is low, water is evaporated through this surface and water contained in this specimen upwardly flowing, then a one dimensional unsaturated flow was formed in this specimen. The evaporation rate at the top surface could be measured by using an evaporation sensor (Watanabe, 1991). When the vapor transportation is one dimensionally transported in the air, the vapor transport rate (evaporation) can be estimated from the absolute humidity gradient that is estimated from the measured relative humidity and temperature. This evaporation rate is the boundary flux condition on the top surface.

\section{Theoretical Model}

The experimental flow system was modeled using the governing equation of a one-dimensional vertical flow in a rigid porous medium as:

$$
\begin{aligned}
& C(\theta) \frac{\partial h}{\partial t}=\frac{\partial}{\partial z}\left(K(\theta) \frac{\partial h}{\partial z}\right) \pm q \\
& h=z+\varphi
\end{aligned}
$$

where, $\theta$ is the saturation, $C=\partial \theta / \partial \mathrm{h}$ is the specific moisture capacity, $K(\theta)$ is the hydraulic conductivity as a function of saturation(In this case homogeneous), $\mathrm{q}$ is the sink or source term, $\mathrm{z}$ is the height from a reference level, $\psi$ is the capillary pressure head, and t is time. Equation (2) can be solved for appropriate initial and boundary conditions and given the $C(\theta)$ and $K(\theta)$ rclations of the medium constitute the direct problem, and vertical saturation distribution can be numerically calculated.

The van-Genuchten model (1980) was used to describe the $\mathrm{K}-\theta-\mathrm{h}$ relations as follow;

$$
\begin{aligned}
& K(\theta)=K S S e^{1 / 2}\left\{1-\left(1-S e^{1 / m}\right)^{m}\right\}^{2} \quad(4) \\
& C(\theta)=\alpha(n-1)(\theta s-\theta d) S e^{1 / m}\left(1-S e^{1 / m}\right)^{m}
\end{aligned}
$$

$$
\begin{array}{lc}
S e=\frac{\theta-\theta d}{\theta s-\theta d} & (0 \leq S e \leq 1) \\
S e=(1+|\alpha \varphi| n)^{-m} & (\alpha>0) \\
n=1 /(1-m) & (0<m<1, n>1)
\end{array}
$$

where, $K s$ is the saturated hydraulic conductivity $(\mathrm{cm} / \mathrm{sec}$.), $\mathrm{Se}$ is the effective saturation, $\theta d(\mathrm{~L} 3 /$ L3 ) and $\theta \mathrm{s}$ ( L3/ L3 ) are the residual and maximum saturation and $\alpha\left(\mathrm{L}^{-1}\right), n$ and $m$ are empirical parameters. When the unsaturated hydraulic properties are modeled, Eq. (2) can be numerically solved for $\theta$ and $h$.

The unsaturated flow program was adapted to include soil saturation data in the objective function. In this program, the least squares parameter optimization is based on Marquardt's maximum neighborhood method [Marquardt, 1963]. The objective function to be minimized in the revised algorithm is:

$$
O(F) \sum_{K=1}^{L}\left\{W_{k}[\theta o(d k)-\theta c(d k, F)]\right\}^{2}
$$

where, $F$ is a vector containing the optimized parameters $\boldsymbol{\alpha}, m, \boldsymbol{\theta} r, \boldsymbol{\theta} s$ in the unsaturated hydraulic properties model, $\theta$ is the saturation at a depth $d k$ through the reference soil profile, the subscripts $o$ and $c$ denote observed and predicted values, $k$ is the measured index, and $L$ is the number of soil moisture measurements over the soil profile. The variable $W$ is weighting coefficient. It was set to $W=1$.

\section{Results and Discussion}

In selecting a site for nuclear waste disposal, it is of much importance to consider the ways on which the leakage will occur. The most important vector of waste migration is groundwater circulating through the surrounding medium. So, among of several studies on the site around the location of the disposal facility in Rokkasho low level waste disposal site, the accurate estimation of unsaturated hydraulic properties is essential for analyzing the rain water seepage to groundwater and the time required for its journey to reach the groundwater passing on the disposal facility.

During the period from Aug. 18 to Aug. 24, 1997, an in-situ test was carried out at a place in Rokkasho low level disposal site on a volcanic ash soil block. The soil block was prepared as in sec. 3, Fig. 1. The initial saturation distribution $\theta I$ was measured by collecting samples from the trench around the soil block. After the test was finished, soil samples were taken for the measurements of final saturation distribution $\theta \dot{o}$, saturated hydraulic conductivity $K s$, dry density $\gamma d$,specific weight of solids Gs, and porosity calculations $\phi$. Also an undisturbed and disturbed soil specimen $(8 \mathrm{~cm}$ diameter, $\& 30 \mathrm{~cm}$ 

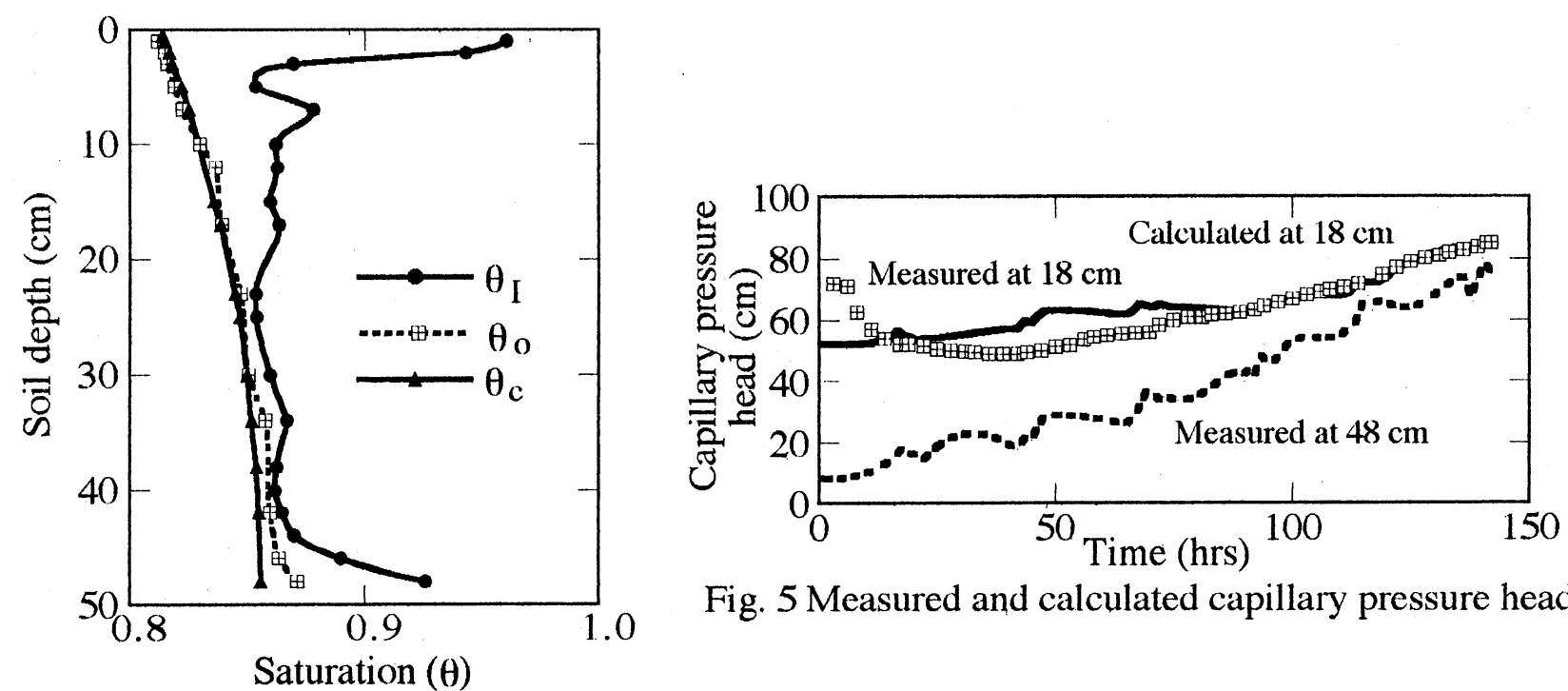

Fig. 5 Measured and calculated capillary pressure head

Fig. 3 In-situ measured and calculated saturation of Volcanic ash.

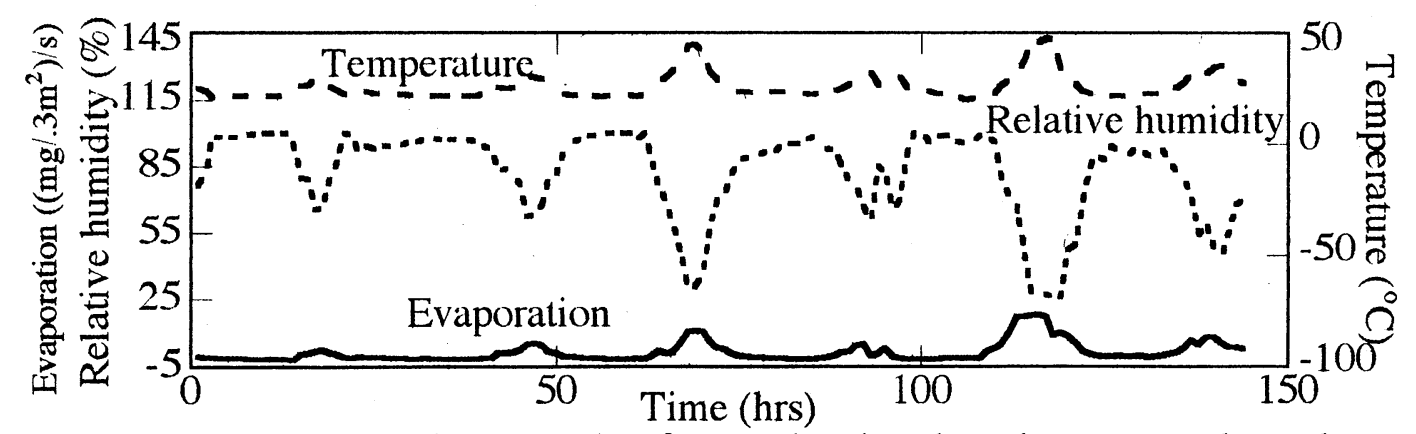

Fig. 4 In-situ measured evaporation from volcanic ash under measured weather condition of air temperature and relative humidity at Rokkasho site.

long) were taken from the soil block for the laboratory test (Fig. 2). The measured evaporation at the top surface and capillary pressure at the bottom of the block were used in the inverse solution with the assumed model parameters $\left(\alpha, \mathrm{m}, \theta_{\mathrm{r}}\right.$ and $\left.\theta \mathrm{s}\right)$ to calculate the final saturation distribution $\theta \mathrm{c}$. Each calculated distribution was compared with the measured one at the end of the test till we reached to a good agreement with different assumption of model parameters.

Figure 3 shows a comparison between the measured and calculated saturation distribution at the beginning and at the end of the test, the objective function $O(F)=0.0013$ in the comparison between $\theta$ $\mathrm{o}$ and $\theta_{\mathrm{c}}$ for estimating the parameters shown in Table 1, where $L=15$ in Eq. (9). From this figure one can observe the effect of evaporation on the soil moisture content, especially in the upper part. Figure 4 is the measured evaporation under the shown weather condition of air temperature and relative humidity; it is noticeable that the measured evaporation is much affected by the temperature and relative humidity of the injected air. Figure 5 shows a comparison between the measured capillary pressure at the bottom of soil block and at $18 \mathrm{~cm}$ depth, and also between calculated capillary pressure at $18 \mathrm{~cm}$ using the estimated unsaturated model parameters shown in Table 1. From this figure and Fig. 3, we can say there is a good agreement between soil dryness and the measured capillary pressure at the two locations. From the comparison between the measured and calculated capillary pressure at $18 \mathrm{~cm}$ depth we can confirm on the accuracy of the proposed unsaturated hydraulic properties estimation technique. The discrepancy between the measured and calculated capillary pressure at the first hours may due to the time required for the tensiometer to reach equilibrium, or to the time required for the downward moving unsaturated flow level to reach the tensiometer level, $(18 \mathrm{~cm})$.

A comparison has been made between the estimated $h(\boldsymbol{\theta})$ and $K(\boldsymbol{\theta})$ relations for volcanic ash, with those measured by Japan Nuclear Fuel Limited. From this comparison (Fig. 6), the good agreement between measured and estimated values confirmed the accuracy of the proposed technique at saturation $0.6-1.0$, and focused on the difficulty in the $h(\theta)$ and $K(\theta)$ measurement to cover a wide rang of the saturation where the measurements were conducted in a narrow saturation range. Also from this figure, 
Table 1 . Soil characteristics and the estimated parameters in van- Genuchten under different condition

\begin{tabular}{|c|c|c|c|c|c|c|c|c|c|}
\hline soil & condition and test & sile & $\gamma_{d} \mathrm{~g} / \mathrm{cm}^{3}$ & $G_{s} \mathrm{~g} / \mathrm{cm}^{3}$ & $K_{S} \mathrm{~cm} / \mathrm{sec}$ & $\alpha$ & $m$ & $\theta_{r}$ & $\theta_{s}$ \\
\hline \multirow{3}{*}{$\begin{array}{l}\text { Kanto- } \\
\text { loam }\end{array}$} & undist., in-situ & Saitama & 0.548 & 2.49 & $2.5 \mathrm{E}-3$ & 0.012 & $(0.449$ & 0.25 & 0.99 \\
\hline & dist., laboratory & Univ. & 0.56 & 2.47 & 3.6E-3 & 0.0119 & 0.4486 & 0.22 & 0.97 \\
\hline & dist., mcasured & Tsukuba & 0.535 & 2.4 & $2.1 \mathrm{E}-3$ & \multicolumn{4}{|c|}{ Mcasured hydraulic conduclivity } \\
\hline \multirow{3}{*}{$\begin{array}{l}\text { Volca- } \\
\text { nic ash }\end{array}$} & undist., in-silu & \multirow{3}{*}{$\begin{array}{l}\text { Rokka- } \\
\text { sho }\end{array}$} & 0.84 & 2.61 & $1.18 \mathrm{E}-4$ & 0.01167 & 0.327 & 0.33 & 0.97 \\
\hline & undist., laboratory & & 0.853 & 2.58 & $1.18 \mathrm{E}-4$ & 0.0117 & 0.327 & 0.35 & 0.96 \\
\hline & $\begin{array}{l}\text { dist., laboratory } \\
\text { undist., measured }\end{array}$ & & $0.8(2)$ & $\begin{array}{l}2.54 \\
2.69\end{array}$ & $\begin{array}{l}8.12 \mathrm{E}-4 \\
1.1 \mathrm{E}-3\end{array}$ & $\begin{array}{l}0.0307 \\
\text { Measured }\end{array}$ & $\begin{array}{l}0.456 \\
\text { hydrauli }\end{array}$ & $\begin{array}{l}0.28 \\
\text { c prope }\end{array}$ & $\begin{array}{l}0.99 \\
\text { rtics }\end{array}$ \\
\hline
\end{tabular}
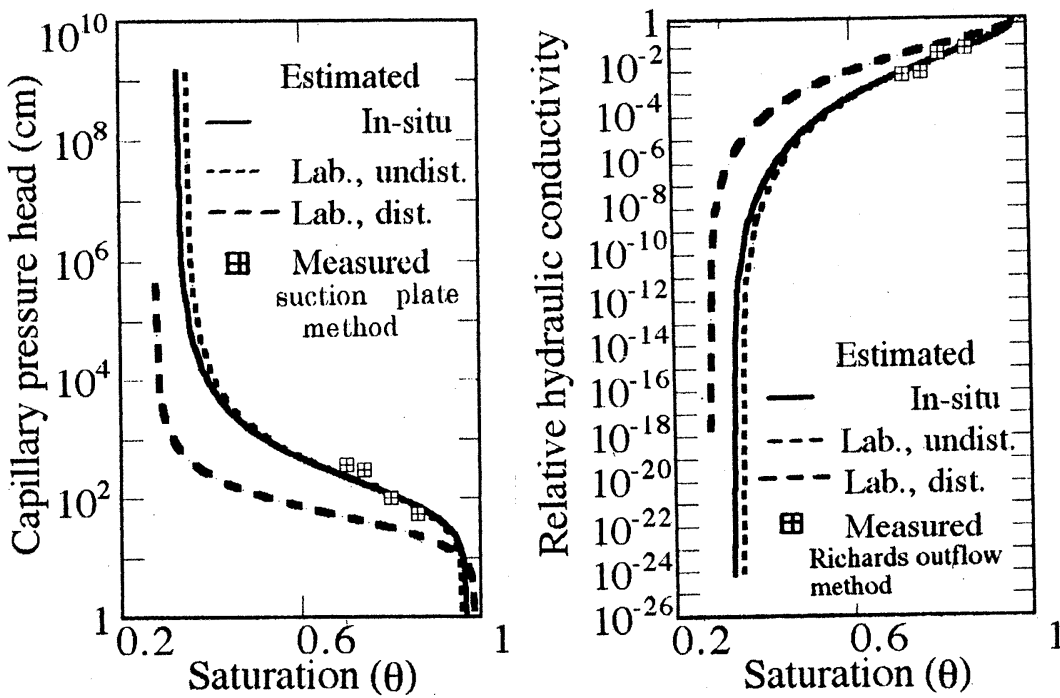

Fig. 6 Measured and estimated unsaturated hydraulic properties of volcanic ash as in Rokkasho low level disposal site.

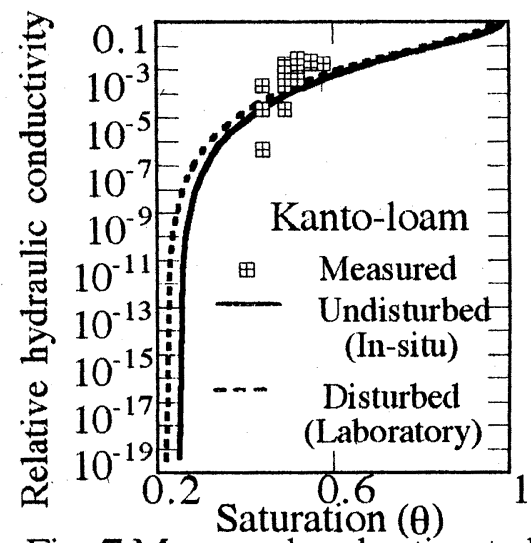

Fig. 7 Measured and estimated hydraulic conductivity.
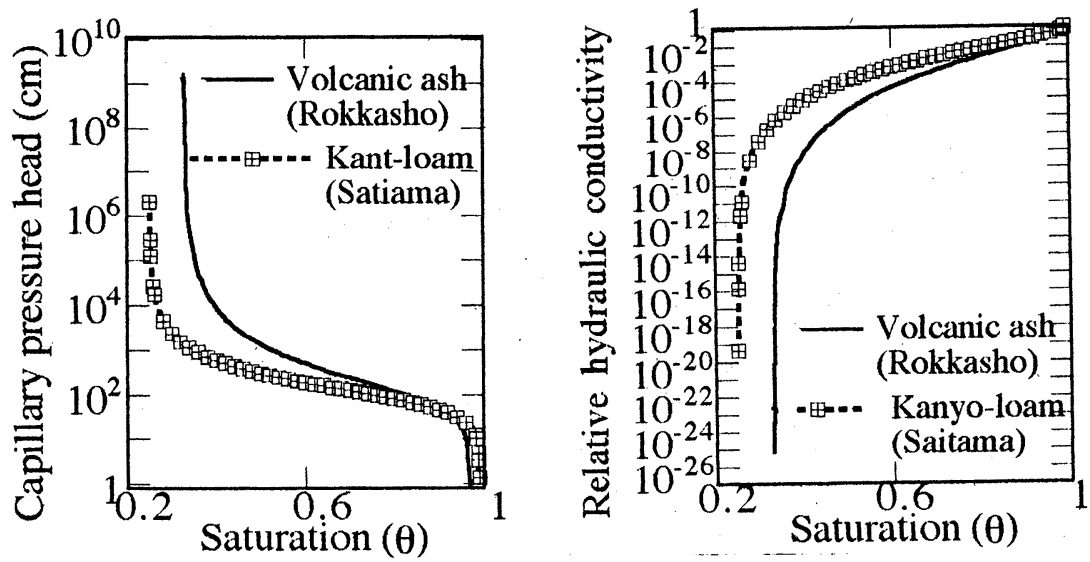

Fig. 8 Comparison between the estimated unsaturated hydraulic properties of volcanic ash in Rokkasho and Kanto-loam type.

the comparison between the estimated unsaturated hydraulic properties from in-situ test, and laboratory tests on undisturbed and disturbed soil specimens reveals that, the laboratory test can replace of the insitu test with a care must be taken when preparing the undisturbed soil specimen, but the case of laboratory disturbed specimen shows the effect of like soil tillage (disturbance) on the soil properties. By the same manner, in-situ and laboratory testes were carried out on the type of volcanic ash found in Saitama University namely by Kanto-loam. The measured soil characteristics and the estimated parameters in van-Genuchten model under different condition of the soil are shown in Table 1. Figure 7 shows the measured unsaturated hydraulic conductivity by Setiawan and Nakano, (1993) in the institute of agricultural and environment, Tsukuba on disturbed Kanto-loam and the estimated values from the in-situ undisturbed and laboratory undisturbed and disturbed soil specimens. The scattering in the measured values for the same saturation may due to the disturbance of soil specimen and the method of packing it, but in general it has an agreement with the estimated values. Shown in Fig. 8 is a comparison 
between the in-situ and laboratory estimated $h(\boldsymbol{\theta}) \operatorname{and} K(\boldsymbol{\theta})$ relations for both types of volcanic ash found in Rokkasho and that in Kanto area (Kanto-loam). From this figure with the aid of soil characteristics in Table 1, we can observe that, the volcanic ash found in Rokkasho is denser (from the values of $\gamma \mathrm{d}$ and $\mathrm{Gs}$ ), and more compacted (from the values of the porosity $\phi$, and $K s$ ), also the soil has high matrix head (from the value of $\alpha$ and from $h(\theta)$ relation) and low unsaturated hydraulic conductivity than the Kanto-loam type.

\section{Conclusions}

The unsaturated hydraulic properties are well estimated using the proposed estimation technique, and the technique seems to be accurate, simple and time effective. From the comparisons between studied two types of volcanic under different condition one can conclude; 1) The laboratory test on undisturbed soil specimen can replace the in-situ test with care must be taken during specimen preparation, 2) The volcanic ash found in Rokkasho is denser, and has high matrix head and low saturated and unsaturated hydraulic conductivity than Kanto-loam, and 3) soil like tillage (disturbance) has much effect on its physical and hydraulic properties.

\section{References}

1) Durner, W., (1994): Hydraulic conductivity estimation for soils with heterogeneous pore structure. Water Resource. Res., 30, 211-223.

2) Marquardt, D. W., (1963): An algorithm for least- squares estimation of non- linear parameters. J. Soc. Indust. appl. Math., 11, 431- 441.

3) Mualem, Y., (1976): A new model for predicting the hydraulic conductivity of unsaturated porous media. Water Resour. Res., 12, 513-522.

4) Ross, P. J., and K. R. J. Stmettem, (1993): Describing soil hydraulic properties with sums of simple functions. Soil Sci. Soc. Am. J., 57, 26-29.

5) Ross, P. J., J. Williams, and K. L. Bristow, (1991): Equation for extending water-retention curves to dryness. Soil Sci. Soc. Am. J., 55, 923-927.

6) Rossi, C., and J. R. Nimmo, (1994): Modeling of soil water retention from saturation to oven dryness. Water Rescuer. Res., 30, 701-708.

7) Setiawan, B. I., and M. Nakano, (1993): On the determination of unsaturated hydraulic conductivity from soil moisture profile and from water retention curves. Soil Sci., 156, 389-395.

8) van-Genuchten, M. T., (1980): A closed-form equation for predicting the hydraulic conductivity of unsaturated soils. Soil Sci. Soc. Am. J., 44, 892-898.

9) Watanabe, K., (1991): Evaporation measurement in validation drift. Part 1, Stripa Tech. Report, 1-131.

10) Watanabe $K$. and $Y$. Tsutsui, (1994): Anew equipment used for measuring evaporation in a field Proc. 7th. Congr. IAEG: 309-313.

(Received September 30,1997) 